\title{
FACTORS ASSOCIATED WITH FOOD SAFETY KNOWLEDGE AND PRACTICE AMONG STREET FOOD VENDORS IN TAUNGGYI TOWNSHIP, MYANMAR: A CROSS-SECTIONAL STUDY
}

\author{
Tin Aung Soe Htway ${ }^{1,2}$ and Kraiwuth Kallawicha ${ }^{1}$ \\ 1 College of Public Health Sciences, Chulalongkorn University, Bangkok 10330, Thailand. \\ 2 Department of Food and Drug Administration, Ministry of Health and Sport, Yangon, Myanmar \\ Corresponding author: Kraiwuth Kallawicha \\ Email: kraiwuthk@gmail.com
}

\begin{abstract}
Food safety is a growing public health concern worldwide. Street foods are an integral part of many cultures and offer at affordable prices. However, it is associated with food safety issues, especially in developing countries. Therefore, it is essential that street food vendors understand and implement food safety practices to prevent outbreaks of foodborne illnesses. This cross-sectional study investigated the food safety knowledge and practices as well as their associated factors among street food vendors in Taunggyi Township, Myanmar. Validated structured questionnaires were used to interview 158 street food vendors, and a set of observational checklists was used to inspect the sanitary conditions of vending sites and vendors' food hygiene practices. The association between food safety knowledge and practices was assessed using a chi-squared test. Our results revealed that most vendors had a high level of food safety knowledge and that $58.9 \%$ scored equal to or lower than the median value in food safety practices, whereas $41.1 \%$ scored higher than the median value (median=15). Sex and education level were significantly associated with participants' food safety knowledge $(p<0.001)$. Similarly, education, race and monthly income were significantly associated with their food safety practices $(p<0.001)$, whereas food safety training attendance had no association. Additionally, participants with better knowledge scores were more likely to have better practice scores. Food safety training given to vendors should be more detailed and comply with standard guidelines, especially with regard to street food safety practices.
\end{abstract}

Keywords: Food safety; street food; knowledge and practices; Myanmar

\section{INTRODUCTION}

Food safety and hygiene are associated with population health and well-being and have consistently remained a major global health challenge, receiving great attention as food-borne diseases persist. The United Nations" "Sustainable Development Goals," particularly goals three and six, focus on health and well-being as well as clean water and sanitation, respectively ${ }^{1,2}$. Foodborne diseases represent a growing public health problem in both developed and developing countries. Annually, millions of individuals worldwide, especially those under 5 years, are hospitalized or die as a consequence of consuming unhygienic foods ${ }^{3,4}$. The World Health Organization (WHO) has reported that food borne illness is a major international health concern and a leading cause of declining economic growth ${ }^{5}$. In a fast paced and rapidly growing society, the majority of the population struggle with daily selfcare. Health promotion behaviors are neglected. Many people consume only non-nutritious readyto-eat food, undercooked food, unhygienic food, and sweetened beverages ${ }^{6}$. In numerous cities, families rely solely on street foods, which are often not prepared in compliance with food safety standards. Currently,78\% of world healthcare expenditures are for the treatment of chronic diseases that many be partially related to diet ${ }^{7}$.
The preparation of street food is a major food safety concern. According to WHO, street foods are normally prepared under unhygienic conditions and sold at sites where sanitation services, safe water, and garbage disposal facilities are limited $^{8}$. Unhygienic conditions at food handling and vending sites are major risk factors for food-borne diseases. Concerns over street food vendors' food safety knowledge and practices have recently become a public health focus. As a result, the assessment of street food vendors' knowledge of food safety guidelines and practices is necessary to identify any gaps vendors may have in their understanding and improve national food safety standards.

In Myanmar, diarrhea is the main cause of morbidity in children under 5 years and is the fourth leading cause of death in children?. Improper food handling by vendors, which arises from either (1) a lack of knowledge about foodborne diseases, (2) a lack of perception of the impact of unhygienic food handling, or (3) inability to change behavior, which has been confirmed as a common cause of food-borne illnesses ${ }^{10}$. In 2018, 37 food poisoning events affecting 1,320 people and 17 diarrheal events affecting 567 people were reported in Myanmar. Among these cases, 1 death from food poisoning and 11 from diarrheal diseases were recorded ${ }^{11}$. Moreover, according to Taunggyi's 2017 health 
profile, a total of 2,342 diarrheal cases ( 5 deaths) and 263 dysentery cases were reported; these conditions ranked first and fourth out of the five leading causes of morbidity and mortality. Moreover, 42 cases of food poisoning were reported $^{12}$.

Taunggyi is the fourth most populous city in Myanmar. The city has a vibrant population of locals, migrants, and tourists who enjoy street food stalls offering famous traditional foods daily. However, the hygiene conditions of street food there is considered poor, which may result from vendors' lack knowledge about safe food handling practices as suggested by the Food and Agriculture Organization (FAO) ${ }^{13}$. Therefore, it is crucial to understand the current situation regarding food safety knowledge and practice among street food vendors to prevent further outbreak of food-borne diseases. In this study, we assessed the level of food safety knowledge and practices of street food vendors in Taunggyi Township and also identified their associated factors. We hope this will help to fill any current regulatory gaps and lead to the development, promotion and implementation of appropriate policy and strategies sustainable safety guidelines, standards, and training for street food vendors.

\section{MATERIALS AND METHODS}

\section{Study design and population}

The study design was cross-sectional study design and data collection was performed between June and July 2020 in Taunggyi Township, eastern Myanmar, which has a population of 386,917 in $2018^{14}$.

The study included all street food stalls that registered with the Taunggyi Municipal Department before the study started (March 2020). We selected one street food vendor from one street food stall who was older than 18 years, mainly cooked and prepared food and were also able to read or speak the official language of Myanmar for the study. After participant agreed to participate in the study, face-to-face interviews with validated structure questionnaires related to food safety knowledge were conducted. The working behaviors and conditions of vending sites were observed by the researcher who followed an observational checklist and not disturbed the vendor's work. Participants were freely to do their work and refuse to participate whenever they feel uncomfortable with the observation. The completed questionnaires were labeled and coded for further statistical analysis.

The study protocol was approved by the University of Public Health-Institutional Review Board (UPHIRB), Ministry of Health and Sports, Republic of the Union of Myanmar. The study was conducted with the permission of Taunggyi Municipal Department and Food and Drug Administration
(FDA). Signed informed consents were obtained from each participant.

\section{Assessment of knowledge level}

Knowledge related to food safety was analyzed using validated structured questionnaires $(\mathrm{IOC}=0.85$, Kuder-Ridcharson $20=0.63)$ which were modified from previous study of Wai and Suksaroj (2018) and WHO guidelines ${ }^{15,16}$. In total, 21 questions were asked, divided into three parts: including (1) knowledge about food-borne diseases and their symptoms, such as diarrhea, dysentery, food poisoning, hepatitis $A$, and typhoid; (2) questions related to street food vendors' personal hygiene practices, such as hand washing, glove use, and hand towel use; and (3) questions related to food handling, preparation and storage practices, such as cleaning of surfaces and food storage.

\section{Assessment of practice level}

Food safety practices were assessed using the observational checklist, which was adopted from the "Basic steps to improve safety of streetvended food" published by $\mathrm{WHO}^{16}$ and $\mathrm{FAO}^{17}$ as well as the "Regional Code of Hygienic Practice for Street Vended Foods in Asia" as outlined by Codex Alimentarius ${ }^{18}$. Observation of each street food stall was conducted by the researcher one by one. In total, 23 items were divided into Part I (environmental hygiene), Part II (personal hygiene), and Part III (food handling, storage, and preparation hygiene).

\section{Sociodemographic variables}

Data on socioeconomic characteristics and food safety training were collected using structured questionnaires. Socioeconomic characteristics included age, sex, education level, ethnic group, and monthly income. Confirmation on food safety training required proof, including date of training, name of the training organization, and training certificates. The sanitary conditions of vending sites were measured using the observational checklist, which contained a total of 23 items. The hygiene conditions of each stall were determined based on distance between the stall and a dump site or toilet facilities, evidence of disease vectors, water supply, and the presence or absence of a wash basin, soap, and towels.

\section{Statistical analysis}

Descriptive statistics were used determined all participants' sociodemographic characteristics and explain their work activities, working conditions, food safety knowledge, and training experience in street food safety.

The food safety knowledge and practices of street food vendors were categorized into levels. Knowledge levels were categorized according to Bloom's cut-off point into three levels: low $(<60 \%)$, medium $(60 \%-80 \%)$, and high (>80\%) (Rahman et al., 2016). Food safety practice level was categorized into two levels: lower or equal to the median and higher than the median. 
The knowledge and practice scores were not normally distributed as it has been tested using Kolmogorov-Smirnov test, thus, the associations between knowledge and practices of food safety guidelines and other variables were evaluated using non-parametric chi-squared test and Fisher's exact test. The associated variables were further evaluated using multiple logistic regression to control the cofounding factors. Statistical significance was indicated by a $p$ value of $<0.05$. All statistical analyses were performed using IBM SPSS Statistics for Windows, version 25 (IBM Corp., Armonk, NY, USA).

\section{RESULTS}

In total, 158 street food vendors registered with the Taunggyi Municipal Department and data were collected from all of these stalls. Table 1 represents participants' sociodemographic characteristics including age, sex, education, race, monthly income and training status. Most participants were male $(60.8 \%)$, and the average age was $36.8 \pm 7.1$ years. Middle-aged adults (age: 36-55 years) were the dominant group $(68.4 \%)$. Although Taunggyi is considered the traditional territory of the Shan ethnic group, the results of this study indicated that the participating vendors were predominantly Burmese (39.2\%). The monthly income of most participants was greater than 400,000 Myanmar Kyats (approximately US\$266).

Among the 158 participants, 30 (19\%) of them attended a food safety training course conducted by the FDA and $10(6.3 \%)$ attended training offered by other organizations. The other 128 (81\%) participants reported that they had never attended any food safety training. The detailed information and characteristics of participants are listed in Table 1.

Table 1 Sociodemographic status of participants $(N=158)$

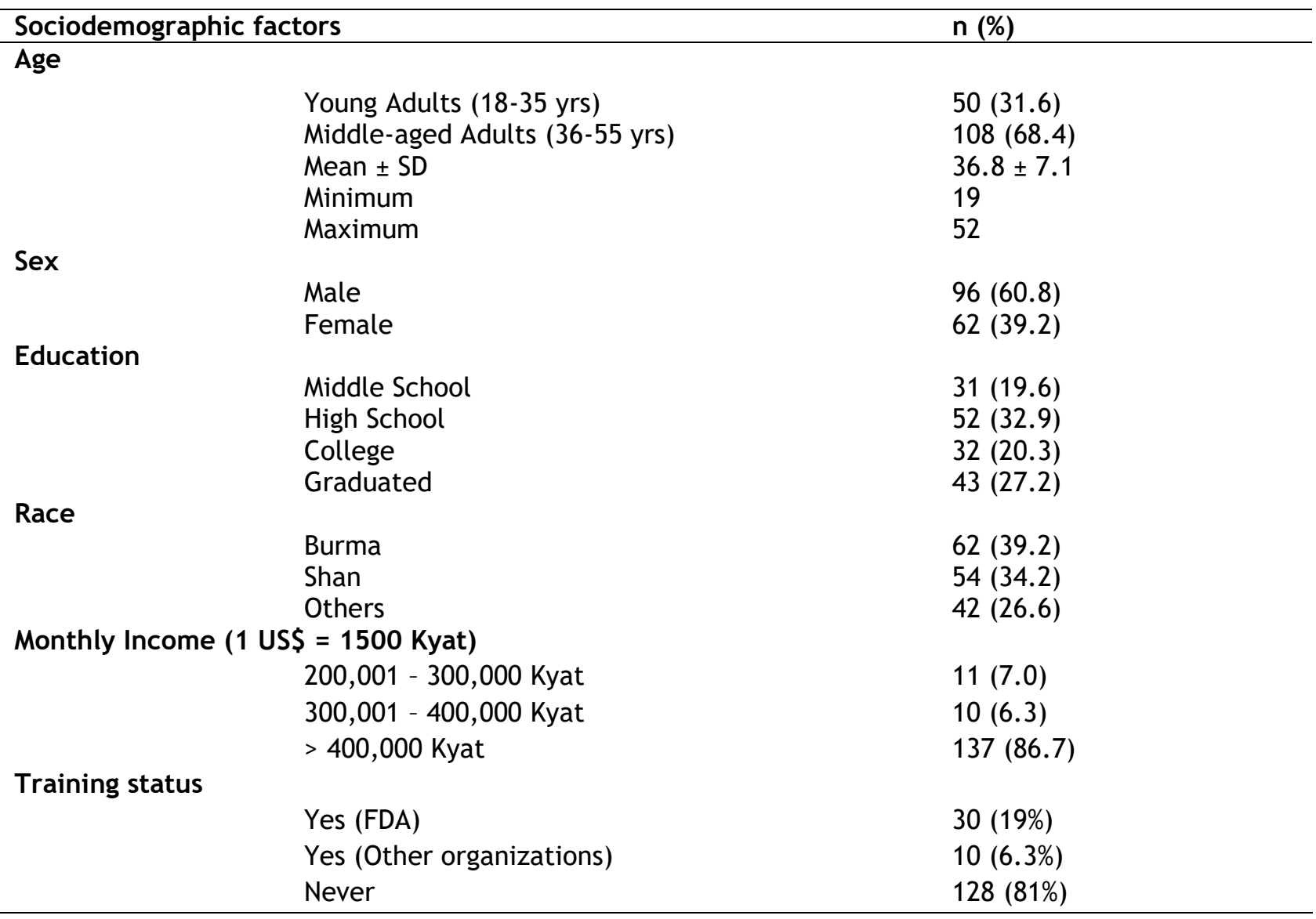

We assessed food safety knowledge by interviewing participants using a structured questionnaire that consisted of 21 questions and grading the answer. Knowledge scores ranged from 16 to 21 , with a mean score of $19.9 \pm 1.4$ (mean \pm SD). Figure 1 represents the level of participants' food safety knowledge. Ten participants $(6.3 \%)$ had moderate levels of knowledge and 148 (93.7\%) participants had high knowledge levels. None of participants had poor knowledge.
The researcher evaluated food safety practices based on observations using a checklist. The food safety practice scores of the participants ranged from 10 to 21 , and the median score was 15 (IQR= $3)$. Figure 2 shows the food safety practice level of participants. Ninety-three (58.9\%) participants had practice scores less than or equal to the median, and 65 (41.1\%) scored higher than the median. 


\section{Level of Knowledge of the Participants}

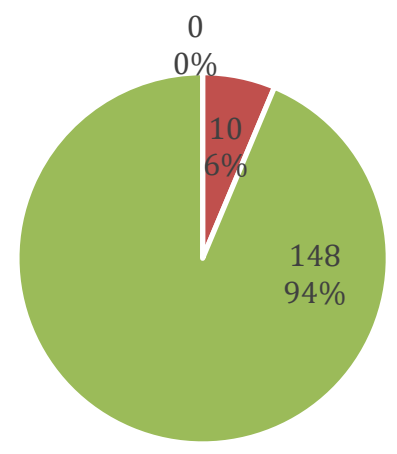

- Poor $(<60 \%)$

- Moderate (60-80\%)

- Good $(>80 \%)$

Figure 1 Distribution of food safety knowledge among street food vendors

\section{Level of Practice of the Participants}

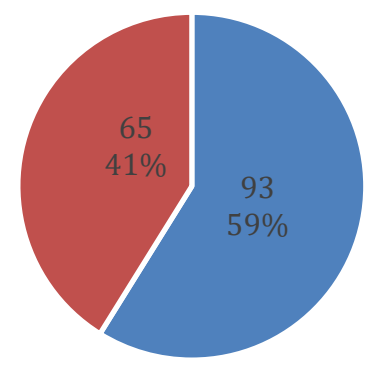

- $\leq$ Median (15)

- > Median

Figure 2 Distribution of food safety practice scores among street food vendors.

Table 2 represents the association analysis of participants' food safety knowledge and sociodemographic factors which revealed that sex, education level, and ethnic group were associated with food safety knowledge $(p<$ 0.001). For this analysis, patients were recategorized based on their food safety knowledge levels $(\leq 80 \%$ and $>80 \%$ ) because no participant fell into the poor knowledge category.

Table 3 shows an association analysis of food safety practices and sociodemographic factors which also revealed that education level, ethnic group, and monthly income were associated with food safety practices $(p<0.001)$.
Additionally, the associations between food safety training and food safety knowledge and practices were analyzed. However, no association was observed. Table 4 presents the association between the food safety knowledge and practices of the participants. Our data revealed a significant association between food safety knowledge and practice of the respondents $(p \leq$ 0.05 ) because participants with better knowledge scores tended to have corresponding practice scores. We further evaluated the association between food safety knowledge and practice and significant variables using the chi-squared test with multiple logistic regression. However, none of the variables exhibited statistical significance in the regression model. 
Table 2 Association between food safety knowledge and sociodemographic factors

\begin{tabular}{|c|c|c|c|c|c|}
\hline \multirow[b]{2}{*}{ Sociodemographic factors } & \multicolumn{2}{|c|}{ Knowledge } & \multirow[b]{2}{*}{$x^{2}$} & \multirow[b]{2}{*}{$d f$} & \multirow[b]{2}{*}{$p$ value } \\
\hline & $\begin{array}{l}\leq 80 \%, \\
\text { n (\%) }\end{array}$ & $\begin{array}{l}>80 \%, \\
\text { n (\%) }\end{array}$ & & & \\
\hline \multicolumn{6}{|l|}{ Age } \\
\hline Young Adults & $2(20.0)$ & $48(32.4)$ & \multirow{2}{*}{0.669} & \multirow{2}{*}{1} & \multirow{2}{*}{0.506} \\
\hline Middle-aged Adults & $8(80.0)$ & $100(67.6)$ & & & \\
\hline \multicolumn{6}{|l|}{ Sex } \\
\hline Male & $0(0)$ & $96(64.9)$ & \multirow{3}{*}{16.530} & \multirow{2}{*}{1} & \multirow{2}{*}{$<0.001$} \\
\hline Female & $10(100)$ & $52(35.1)$ & & & \\
\hline \multicolumn{5}{|l|}{ Education } & \\
\hline Middle School & $0(0)$ & 31 (20.9) & \multirow{4}{*}{21.762} & \multirow{4}{*}{3} & \multirow{4}{*}{$<0.001$} \\
\hline High School & $10(100)$ & $42(28.4)$ & & & \\
\hline College & $0(0)$ & $32(21.6)$ & & & \\
\hline Graduated & $0(0)$ & $43(29.1)$ & & & \\
\hline \multicolumn{6}{|l|}{ Ethnic group } \\
\hline Burma & $10(100)$ & $52(35.1)$ & \multirow{3}{*}{16.530} & \multirow{3}{*}{2} & \multirow{3}{*}{$<0.001$} \\
\hline Shan & $0(0)$ & $54(36.5)$ & & & \\
\hline Others & $0(0)$ & $42(28.4)$ & & & \\
\hline \multicolumn{6}{|c|}{ Monthly Income (1 US\$ = 1500 Kyat) } \\
\hline $200,001-300,000$ Kyat & $0(0)$ & $11(7.4)$ & \multirow{3}{*}{1.636} & \multirow{3}{*}{2} & \multirow{3}{*}{1.000} \\
\hline $300,001-400,000$ Kyat & $0(0)$ & $10(6.8)$ & & & \\
\hline$>400,000$ Kyat & $10(100)$ & $127(85.8)$ & & & \\
\hline
\end{tabular}

Note: Fisher's exact test with significance denoted by $p<0.05$

Table 3 Association between food safety practice and sociodemographic factors

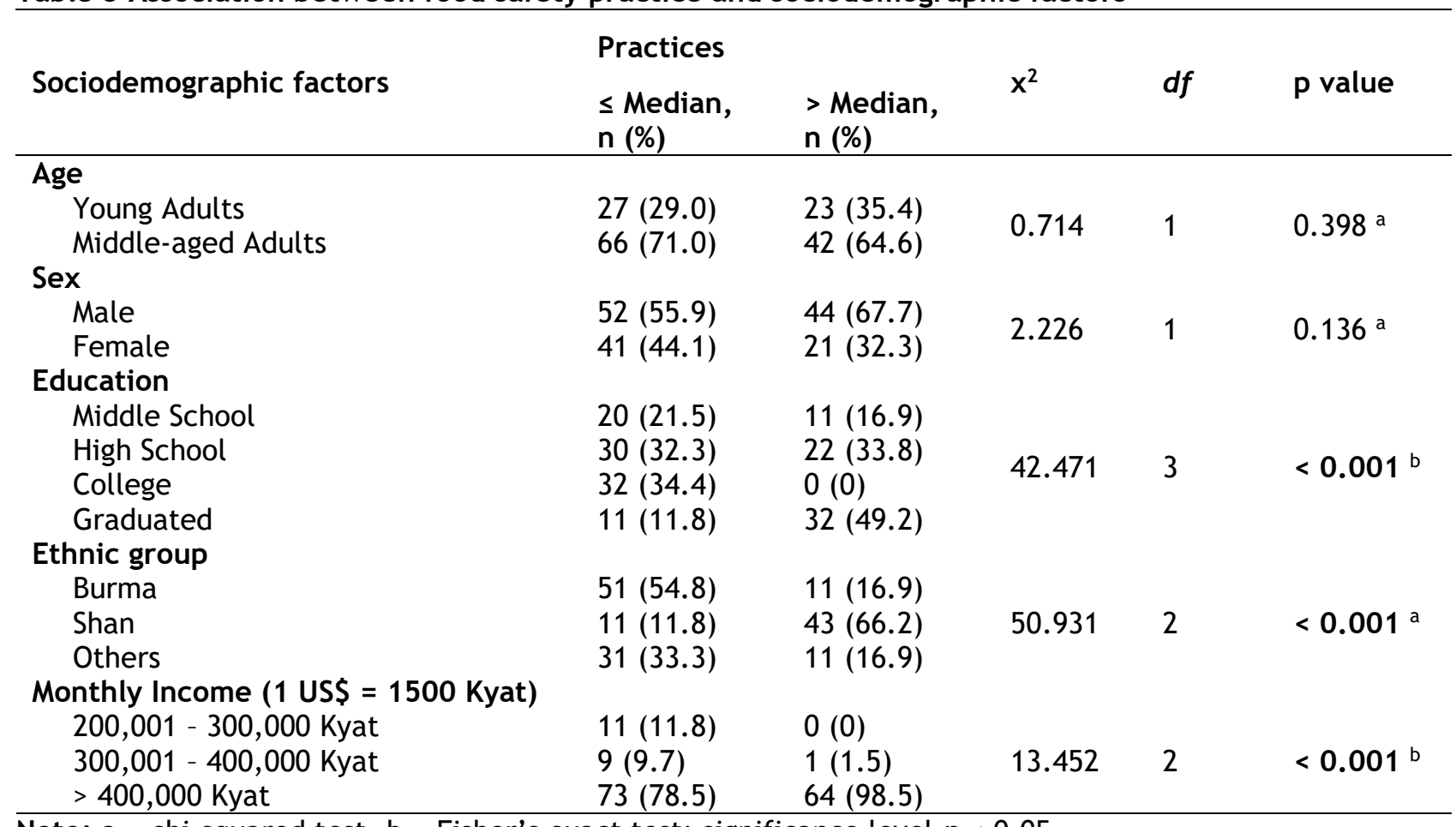

Note: $\mathrm{a}=$ chi-squared test, $\mathrm{b}=$ Fisher's exact test; significance level $p<0.05$

Table 4 Relationship between food safety knowledge and practices

\begin{tabular}{|c|c|c|c|c|c|}
\hline Variables & $\begin{array}{l}\text { Practices } \\
\leq \text { Median, } \\
\mathrm{n}(\%)\end{array}$ & $\begin{array}{l}\text { > Median, } \\
\text { n (\%) }\end{array}$ & $x^{2}$ & $d f$ & $p$ value \\
\hline $\begin{array}{r}\text { Knowledge } \\
\leq 80 \% \\
>80 \%\end{array}$ & $\begin{array}{l}10(10.8) \\
83(89.2)\end{array}$ & $\begin{array}{l}0(0) \\
65(100)\end{array}$ & 7.461 & 1 & 0.006 \\
\hline
\end{tabular}

Note: Fisher's exact test with significance level $p<0.05$ 


\section{DISCUSSION}

Food safety knowledge and practice are a crucial concern with serious impacts on public health, and unhygienic handling of food could potentially cause major outbreaks of food-borne illnesses. In this study, we assessed the food safety knowledge and practices of street food vendors in Taunggyi Township, Myanmar. The participants had high knowledge scores, with an average score of 19.9 (maximum score: 21 ). Among all vendors, $94 \%$ of them had high knowledge scores ( $>18$ out of total score 21); this proportion is higher than that in a study conducted on vendors in Nay Pyi Taw $(62.5 \%)^{15}$. Compared with another food safety knowledge and practice study in Indonesia, our participants showed better knowledge of food safety $(94 \% \text { vs. } 58 \%)^{19}$. Additionally, Taunggyi Township street food vendors also displayed a better understanding of food safety than the respondents in a food safety study conducted in Malaysia, which found that $36.8 \%$ of participants possessed a good knowledge level ${ }^{20}$. The higher knowledge scores of our participants could be a result of social media, television, and radio campaigns promoting of food safety practices and sharing knowledge. The vendors in our study may have also gained knowledge regarding hygiene practices during the COVID-19 pandemic. Information related to personal hygiene and disease prevention had been extensively distributed during the study period, which may have resulted in higher food safety scores compared with those in other studies conducted prior to the COVID-19 pandemic.

An assessment of food safety practices revealed that $41 \%$ of participants scored higher than the median score of 15, as presented in Figure 3. This poorer score may have arisen from individuals' prioritizing financial incentives over best food safety practices. Lax regulation and enforcement of food safety practices could also contribute to poor score in that area. Although food safety training was implemented and encouraged, food safety inspection and regulations are inadequate. The FDA and local authorities do not closely monitor street food vendors to ensure that they follow practice guidelines. Despite those pitfalls, most participants in our study scored above average compared with those in other studies ${ }^{19,20}$. However, higher score than median does not mean good practice in our study because the practice scores were not normally distributed. It is only implied that the vendor had higher score than most vendors.

Our study revealed that participants' sex, education level, and ethnic group were significantly associated with food safety knowledge. Similar results were also observed in the study of Rahman (2012), who found that Malays people had better knowledge about food safety than non-Malays and that food safety knowledge score is associated with vendors' education level ${ }^{20}$. Similarly, a study in Ho Chi Min City, Vietnam, revealed a significant association between education level and food safety knowledge $^{21}$. In support of this notion, street food vendors in China with a lower education level appeared to be less receptive to learning new hygiene techniques and gaining new food sanitation knowledge ${ }^{22}$. However, some studies among other geographical areas have provided inconsistent results. A study conducted in Guwahati, Assam, India, reported that vendors' education level had no significant effect on their food safety knowledge ${ }^{23}$. Therefore, regionspecific studies are required to better understand food safety problems based on different norms, customs, and cultural features.

Along with education level, our study also demonstrated that participants' ethnic group and monthly income were also significantly associated with their food safety practices. These findings are similar to those from studies conducted in Pakistan and Indonesia, which reported that an association between participants' level of education and food safety practices ${ }^{20,24}$.

This study revealed a significantly positive association between respondents' food safety knowledge and practices. Furthermore, it indicated that street food vendors with a high level of food safety knowledge were likely to apply their knowledge to hygienic practices. Similar findings were obtained in a study conducted in Anbon City Indonesia ${ }^{19}$ and Sarawak, Malaysia ${ }^{20}$. Moreover, a study reported that in Malaysia, food safety knowledge was the most influential factor that contributed to street food vendors exercising good hygiene practices ${ }^{25}$.

Originally, we hypothesized that training experience would be associated with food safety knowledge and practices. However, we did not observe any association between the two in this study, although a study conducted in Nay Pyi Taw did identify such an association ${ }^{15}$. The number of street food vendors $(81 \%)$ who had never attended any food safety training courses may account for this. The percentage of untrained vendors in our study was much higher than in the studies in Ambon City of Indonesia and Johannesburg, South Africa, where $52.6 \%$ and $48.5 \%$ of respondents were untrained, respectively ${ }^{19,26}$. The lack of an association between respondents' food safety training and food safety knowledge and practices scores may be attributed to external factors. For example, training is not mandatory to obtain a sales license from the municipal office. Moreover, it is possible that respondents' training may not have provided sufficient information on food safety practices, consequently resulting in low scores.

Our cross-sectional study analyzed all street food vendors in Taunggyi Township. As a result, this study can be used as a model to reflect the current data on vendors' food safety knowledge 
and practices. This study can prove useful for the local government and community create suitable intervention programs to help ensure sanitary street food conditions and improve related food safety knowledge and practices. Legislators and implementing entities should also use this information to develop realistic strategies to increase compliance with best hygienic practices among street food stalls in Myanmar. One approach could be to close regulatory gaps and establish new street food safety laws and regulations, such as standards, guidelines, and training.

Although this study reflects the current state of street food safety knowledge and practices in Taunggyi Township, it had some limitations. First, this study was limited to Taunggyi Township, Shan State, Myanmar. Results from this study might not reflect food safety knowledge and practices of street food vendors in other parts of the country where geography and culture are different. Second, our study had very limited time of data collection due to COVID-19 lockdown in Myanmar. The researchers and assistants were divided into several teams for data collection. Although, the researchers had been trained for the content of questionnaire and checklist, but inter-observer variation could introduce bias to the results obtained from different observers. Moreover, we did not perform microbial inspections of food samples. Therefore, we could not correlate food safety practices to food hygiene.

\section{CONCLUSION}

Our study found that most vendors have good food safety knowledge, but that their hygienic practices require improvement. Therefore, Food and Drug Administration (FDA), public health department, and Municipal Department should encourage on food safety training with specialized courses according to the global health guidelines for street food vendors. The registration process should mandate that street food vendors provide the local authority with documentation such as current food safety training certificates, medical certificates, and outlines of the basic infrastructure of the basic infrastructure of street food stalls. Moreover, FDA should maintain food safety standard and regulation programs such as monthly or unannounced inspection with regular monitoring and microbial surveillance of street food to improve food hygiene in the food vending industry. Moreover, the local authority should implement the motivational campaigns and food safety advocacy programs that target street food vendors to encourage the use of hygienic practices.

ACKNOWLEDGEMENT The author would like to thank all street food vendors in Taunggyi Township for their contribution to this study.
CONFLICT OF INTEREST: The authors have no conflicts of interests to declares.

\section{REFERENCES}

1. United Nations. About The Sustainable Development Goals. 2015; [Cite 2020 March 20] Available from: https://www.un.org/sustainabledevelop ment/sustainable-development-goals.

2. Velusamy V, Arshak K, Korostynska O, Oliwa K, Adley C. "An overview of foodborne pathogen detection: In the perspective of biosensors." Biotechnology advances. 2010; 28(2): 232-254.

3. World Health Organization (WHO). WHO estimates of the global burden of foodborne diseases: foodborne disease burden epidemiology reference group 2007-2015. World Health Organization. 2015; [Cite 2020 August 31]. Available from:

https://www.who.int/foodsafety/public ations/foodborne_disease/fergreport/en /

4. Osaili, TM, Obeidat BA, Jamous DOA, Bawadi HA. "Food safety knowledge and practices among college female students in north of Jordan." Food Control. 2011; 22(2): 269-276.

5. Mensah P, Yeboah-Manu D, Owusu-Darko K, Ablordey A. "Street foods in Accra, Ghana: how safe are they?" Bulletin of the World Health Organization. 2002; 80: 546554.

6. Samapundo, S., R. Climat, R. Xhaferi and F. Devlieghere (2015). "Food safety knowledge, attitudes and practices of street food vendors and consumers in Port-au-Prince, Haiti." Food Control 50: 457-466.

7. Wardlow I. "How Does Food Impact Health? Taking Charge of Your Health \& Wellbeing."2018; [Cite 2020 May 26]. Available from: https: / /www.takingcharge.csh.umn.edu/ how-does-food-impact-health

8. World Health Organization (WHO). Assuring food safety and quality: guidelines for strengthening national food control systems. 2003; [Cite 2020 August 
31]. Available from: http: / / www.fao.org/3/y8705e/y8705e00 .htm

9. Department of Population. Census Atlas Myanmar, Myanmar population and housing census, Nay Pyi Taw, Myanmar. 2014; Available from: https: //myanmar.unfpa.org/sites/defaul t/files/pub-

pdf /MyanmarCensusAtlas_lowres.pdf

10. Waiyeelinn. Integrated Food Control Systems Toward Food-Safety and TradePromotion in Myanmar, Hiroshima University. 2016.

11. Central Epidermiolgy Unit, Myanmar. Annual Health Report. Department of Public Health. 2018.

12. Shan State Public Health Department. Taunggyi Health Profile. 2017

13. Food and Agriculture Organization (FAO) (1995). "Report of an Food and Agricultural Organization (FAO) technical meeting on street foods, Calcutta, 6-9 November 1995." FAO Food and Nutrition paper 63.

14. Myanmar Information Management Unit (MIMU). "Township Profile 2018"; [Cite 2020 August 31]. Available from: https://themimu.info/townshipprofiles?field_doc_tx_state_regions_tid $=6$ 2

15. Wai KH, Suksaroj T. Food sanitation of salad vending in the markets of Nay Pyi Taw, Myanmar. Journal of Public Health and Development. 2020;18(1):20-38.

16. World Health Organization (WHO) International Food Safety Authorities Network (INFOSAN). Basic steps to improve safety of street-vended food. 2010; [Cite 2020 August 31]. Aailable from:

https: / / www.who.int/foodsafety/fs_man agement/No_03_StreetFood_Jun10_en.p df?ua $=1$

17. Food and Agriculture Organization (FAO). Report of an FAO technical meeting on street foods, Calcutta, 6-9 November
1995. FAO Food and Nutrition paper. 63. [Cite 2020 August 31]. Available from: http: / /www.fao.org/3/W4128T/W4128T 00.htm

18. Codex Alimentarius. Regional Code of Hygienic Practice for Street Vended Foods in Asia. 2017, [Cite 2020 August 31]. Available from: http://www.fao.org/faowho-codexalimentarius/shproxy/en/?lnk=1\&url=https $\% 253 \mathrm{~A} \% 252 \mathrm{~F} \% 2$ 52Fworkspace.fao.org\%252Fsites $\% 252 \mathrm{Fco}$ dex\%252FStandards\%252FCXC\%2B76R2017\%252FCXP_076Re.pdf

19. Sihombing J, Padmawati RS, Kristina SA. "Knowledge, attitude, and practices regarding food safety among food employees in Ambon City, Indonesia." Malaysian Journal of Nutrition. 2018; 24(2): 293-299.

20. Rahman MM, Arif MT, Bakar K, Talib Z. "Food safety knowledge, attitude and hygiene practices among the street food vendors in Northern Kuching City, Sarawak." Borneo Science. 2012; 31; 107116.

21. Samapundo S, Thanh TC, Xhaferi R, Devlieghere F. "Food safety knowledge, attitudes and practices of street food vendors and consumers in Ho Chi Minh city, Vietnam." Food Control. 2016; 70: 79-89.

22. Liu Z, Zhang $G$ and Zhang X. "Urban street foods in Shijiazhuang city, China: Current status, safety practices and risk mitigating strategies." Food Control. 2014; 41: 212-218.

23. Choudhury M, Mahanta L, Goswami J, Mazumder M, Pegoo B. "Socio-economic profile and food safety knowledge and practice of street food vendors in the city of Guwahati, Assam, India." Food Control. 2011; 22(2): 196-203.

24. Ahmed Z, Afreen A, Hassan MU, Ahmad H, Anjum N, Waseem M. "Exposure of food safety knowledge and inadequate practices among food vendors at Rawalpindi; the fourth largest city of Pakistan" Journal of Food and Nutrition Research. 2017; 5(1): 63-73. 
25. Ismail FH, Chik CT, Muhammad R, Yusoff NM. "Food safety knowledge and personal hygiene practices amongst mobile food handlers in Shah Alam, Selangor." Procedia-Social and Behavioral Sciences. 2016; 222: 290-298.

26. Campbell PT. Assessing the Knowledge, Attitudes and Practices of Street Food Vendors in the City of Johannesburg regarding Food Hygiene and Safety. Masters' Thesis. School of Public Health, University of the Western Cape. South Africa. 2011. 